\title{
BMJ Open Cost-utility and budget impact analysis of tocilizumab for the treatment of refractory systemic juvenile idiopathic arthritis in Thailand
}

Nitichen Kittiratchakool, ${ }^{1}$ Disorn Kulpokin, ${ }^{1}$ Chonticha Chanjam, ${ }^{1}$
Soamarat Vilaiyuk, ${ }^{2}$ Sirirat Charuvanij, ${ }^{3}$ Gun Phongsamart, ${ }^{4}$ Parichat Khaosut,
Manasita Tanya, ${ }^{6}$ Ratanavadee Nanagara, ${ }^{7}$ Sira Nantapaisarn, ${ }^{8}$
Pattara Leelahavarong (10) ${ }^{1}$
To cite: Kittiratchakool N, Kulpokin D, Chanjam C, et al. Cost-utility and budget impact analysis of tocilizumab for the treatment of refractory systemic juvenile idiopathic arthritis in Thailand. BMJ Open 2020;10:e037588. doi:10.1136/ bmjopen-2020-037588

- Prepublication history and additional material for this paper are available online. To view these files, please visit the journal online (http://dx.doi. org/10.1136/bmjopen-2020037588).

Received 08 February 2020

Revised 23 April 2020

Accepted 19 July 2020
Check for updates

(c) Author(s) (or their employer(s)) 2020. Re-use permitted under CC BY-NC. No commercial re-use. See rights and permissions. Published by BMJ.

For numbered affiliations see end of article.

Correspondence to Pattara Leelahavarong; pattara.|@hitap.net

\section{ABSTRACT}

Objectives This study aimed to analyse the cost-utility and budget impact of adding tocilizumab to the standard treatment for patients with refractory systemic juvenile idiopathic arthritis (SJIA) in Thailand.

Design Economic evaluation using a decision-analytical model.

\section{Setting Thailand.}

Participants Patients with refractory sJIA who were $\geq 2$ years old.

Methods The use of tocilizumab as an add-on therapy to standard treatment was compared with standard treatment alone. A simulated health state transition model was used to estimate the lifetime costs and health outcomes from a societal perspective. Direct medical costs were collected from tertiary hospital databases while direct non-medical costs were derived from interviews. Health-related quality of life $(Q \circ L)$ was measured using the proxy version of three-level EuroQol five-dimensional questionnaire (EQ-5D-3L). Future costs and outcomes were discounted at an annual rate of $3 \%$. The base case population was patients aged 9.41 years old at refractory disease onset. The results were reported as incremental cost-effectiveness ratios (ICER) in US dollar (USD). One-way and probabilistic sensitivity analysis were conducted to investigate parameter uncertainty. The 5-year budget impact was estimated from a governmental perspective.

Results The ICER of standard treatment plus tocilizumab was US\$35799 per quality-adjusted life-year (QALY) gained compared with standard treatment alone, which was not cost-effective at the threshold of US\$5128 per QALY gained. The estimated 5 years budget impact was approximately US\$4.8 million.

Conclusions The use of standard treatment plus tocilizumab was not cost-effective in the Thai context, which has limited data. However, there is currently no second-line treatment for refractory sJIA in the Thai National List of Essential Medicines; thus, patients must receive higher doses of standard treatment which can cause many side effects. In contrast, tocilizumab showed obvious efficacy in clinical trials in improving treatment response and QoL. Therefore, the price of tocilizumab

\section{Strengths and limitations of this study}

- This multicentre study is the first economic evaluation of tocilizumab use in patients with refractory systemic juvenile idiopathic arthritis (sJIA) in Thailand. Data were collected from tertiary hospitals where paediatric rheumatologists provide treatment to patients with SJIA.

- This study was conducted to inform the subcommittee for the development of the National List of Essential Medicines in Thailand.

- The real-world data were necessary to investigate effectiveness of tocilizumab as an add-on therapy, which might be underestimated.

- The initial Markov model containing functional impairments could not be employed due to the limited data of patients with irreversible Steinbrocker's class III/IV during the data collection period.

should be negotiated to reduce the financial impact on the healthcare system.

\section{INTRODUCTION}

Systemic juvenile idiopathic arthritis (sJIA) is a subtype of JIA, which is related to the predominant abnormality of an innate immune system. However, its pathophysiology and triggers remain unknown. ${ }^{1-3}$ Considering the 2014 International League of Associations for Rheumatology (ILAR) classification criteria, sJIA occurs before the 16 th birthday and persists for at least 6 weeks. The main characteristics include systemic features (ie, quotidian fever, salmon rash, hepatosplenomegaly, lymphadenopathy, peritonitis, pericarditis and pleuritis), arthritis and elevated inflammatory markers. ${ }^{1} \quad 4 \quad 5$ Although the mortality rate of sJIA is unclear, it can lead to life-threatening complications, such as macrophage activation syndrome 
(MAS), massive pericardial effusion and low quality of life (QoL) ${ }^{46-9}$ The epidemiology of sJIA is currently unclear. A systemic review showed that the global prevalence and incidence of JIA were $20.5 / 100,000,95 \%$ CI 19.8 to 21.3 and $7.8 / 100,000,95 \%$ CI 7.6 to 8.1 , respectively, ${ }^{10}$ while the proportions of sJIA to JIA were $5 \%-10 \%$ in North America and Europe, and 25\%-50\% in Asia. ${ }^{11}$ In Thailand, a retrospective study over a 15-year period from 1997 to 2012 in a single referral centre indicated that sJIA contributes to $33.8 \%$ of JIA. ${ }^{12}$

In Thailand, the National List of Essential Medicines (NLEM) is a pharmaceutical reimbursement list used by three main public health schemes: Universal Coverage scheme, Civil Servant Medical Benefit Scheme and Social Health Insurance. ${ }^{13}$ The current version (2019) of NLEM includes six categories. The A, B and C categories are the first-line, second-line and third-line medicines, respectively. The $\mathrm{D}$ category comprises medicines with high costs that are used for specific diseases and must be prescribed by subspecialists and pharmacists who must conduct a drug-use evaluation whenever prescribed. The E(1) category includes medicines under special programmes adopted by the government sector that require monitoring and evaluation, while the $\mathrm{E}(2)$ category includes medicines with a high cost that are used in specific cases, and are restricted in use because they must be prescribed by specific specialists. This medicine group is defined as special-access items that require financial allocation from health insurance schemes to be accessible. $^{14}$

The standard treatment used for sJIA under the NLEM are non-steroidal anti-inflammatory drugs (NSAIDs), systemic corticosteroids and non-biological diseasemodifying antirheumatic drugs (DMARDs). Although these medicines are included in the A-D categories of the Thai NLEM, there are some limitations, including inadequate efficacy in some patients with sJIA and serious side effects, particularly the long-term effects of corticosteroids among children. ${ }^{14}{ }^{15}$ Patients with sJIA who have an inadequate response to standard medicines often progress to refractory sJIA, which requires biological agents for add-on therapy. The American College of Rheumatology (ACR) recommends a variety of biological therapies for sJIA with active systemic features, including interleukin-1 receptor antagonists (anakinra, canakinumab, rilonacept), interleukin-6 receptor antagonists (tocilizumab) and tumour necrosis factor-alpha inhibitors (adalimumab, etanercept, infliximab).

However, tocilizumab is the only biological agent available for treating patients with refractory sJIA in Thailand because of its efficacy, safety, labelled indication and availability. ${ }^{16}$ Unfortunately, tocilizumab can cost more than US $\$ 3846$ for the first 3 months, which may cause financial risks for patients' households. ${ }^{17}$ Moreover, the evidence for the cost-effectiveness of tocilizumab are limited, especially in Thailand. According to our systematic review, five conference abstracts have been published, but no full-text articles are available. ${ }^{18-22}$
Due to the limitations of standard treatment for refractory cases and the obvious efficacy of tocilizumab in terms of inactive disease and improving physical functions and health-related QoL according to clinical trials, ${ }^{23}{ }^{24}$ the Working Group of National Experts' on NLEM selection: orthopaedics and arthrides subgroup proposed moving tocilizumab to the NLEM E(2) category (ie, a special-access item). To that end, the NLEM subcommittee requires both cost-effectiveness and budget impact evidence to inform their decision making. This study aims to analyse the cost-utility and budget impact of tocilizumab used in patients with refractory sJIA as an add-on therapy to standard treatment.

\section{METHODS}

This study was conducted in compliance with the second edition of the Guidelines for Health Technology Assessment (HTA) in Thailand ${ }^{25}$ and the Thai HTA process guideline,${ }^{26}$ which stipulated that research protocols must be considered and approved by the Health Economic Working Group under the NLEM Subcommittee. ${ }^{27}$ Moreover, stakeholders' meetings were organised to consider the methodology and the preliminary results, which included paediatric rheumatologists, rheumatologists, Thai Rheumatism Association, policy-makers, healthcare insurers and representatives of pharmaceutical companies. ${ }^{16} 28$

\section{Study design}

A model-based economic evaluation using a Markov model was developed in Microsoft Excel (Microsoft, Redmond, Washington, USA) to estimate the incremental cost-effectiveness ratio (ICER) in terms of cost per quality-adjusted life year (QALY) with a discount rate of $3 \%$ per annum for both costs and outcomes from a societal perspective. The ICER was then compared with a cost-effectiveness threshold of US $\$ 5128$ per QALY gained. The standard treatment combined with tocilizumab was compared with the existing standard treatment without tocilizumab from a societal perspective. The data for the clinical conditions, health outcomes (ie, life years and QALYs) and costs were collected from seven tertiary hospitals between November 2018 and February 2019 using two main data sources, including medical records and interviews. The clinical conditions data were used for survival analyses to generate transitional probabilities because the treatment options, dosages, regimens, outcome measurements and study population of this study differed from those of clinical studies based on our findings from systematic review (see online supplementary table S1).

\section{Intervention and comparators}

According to the Thai NLEM, the standard treatment or standard medicines used for refractory sJIA are NSAIDs (eg, naproxen, ibuprofen and indomethacin), systemic corticosteroids (eg, prednisolone and 
methylprednisolone) and non-biological DMARDs (eg, sulfasalazine, methotrexate and hydroxychloroquine). In this observational study, tocilizumab as an add-on therapy to standard treatment was compared with standard treatment alone. Generally, tocilizumab was initially prescribed at an $8-12 \mathrm{mg} / \mathrm{kg} /$ dose every 2 weeks, which was subsequently adjusted according to the variety of individual patients' clinical responses. Thus, the cost calculations for the tocilizumab course was designed during the stakeholders' meeting ${ }^{16}$ according to the best practice in the case that tocilizumab was available for every patient who needed it. The patients' weights for dosage calculation were obtained from the weight-for-age in the normative reference data for weight, height, and nutritional indices for children and adolescents in the Thai population (age range, 1 day to 19 years) as applied from the Thai Ministry of Public Health guidelines ${ }^{29}$ Additionally, the patients could receive only one course of tocilizumab in a lifetime. ${ }^{1628}$ In cases of relapse, patients would receive the standard treatment alone with a higher dosage in comparison with the dose for general sJIA treatment.

\section{Target population}

This cost-utility analysis focused on patients with refractory sJIA who were $\geq 2$ years old, who were diagnosed with sJIA according to the ILAR classification of JIA, ${ }^{130}$ and had an inadequate response to the standard treatment, including NSAIDs, systemic corticosteroids and nonbiological DMARDs. ${ }^{16}$ An inadequate response to standard treatment is defined as uncontrollable disease after receiving all of the following therapies: (1) maximum dose of any NSAIDs for at least 1 month; (2) one or more types of non-biological DMARDs in case of sJIA with systemic features, and at least two types of non-biological DMARDs in case of sJIA without systemic features for 6 months and (3) unable to discontinue or reduce dose of systemic corticosteroids to $0.5 \mathrm{mg}$ or less of a prednisone equivalent per kilogram per day following 6 months of treatment. ${ }^{1623}$ In this study, the base case population included patients with refractory sJIA who were aged 9.41 years old when refractory disease occurred. This age was the mean age at refractory disease onset for 43 Thai patients from seven tertiary hospitals (table 1). In addition, a subgroup analysis of patients with refractory disease onset at 15 years old was also employed. This age was chosen because the duration of tocilizumab treatment was around 2 years and the maximum age of patients in the clinical trial was 17 years old. ${ }^{23}$

Considering the epidemiological data in the absence of a national data registry for sJIA in Thailand, the number of patients with refractory sJIA was estimated using several data sources, which were verified by stakeholders' meetings. sJIA occurs before patients are 16 years old ${ }^{1}$ and the labelled indication for use of tocilizumab in Thailand is for patients aged $\geq 2$ years old ${ }^{31}$ while the randomised trial of tocilizumab in sJIA was conducted in patients aged 2-17 years old. ${ }^{23}$ Therefore, the possible target population was Thai people aged 2-17 years old, which
Table 1 Baseline demographic and clinical characteristics of patients with refractory SJIA

\begin{tabular}{|c|c|}
\hline Characteristic & $\mathrm{n}=43$ \\
\hline Male sex-no (\%) & $21(48.83)$ \\
\hline Age-years old (SD) & $9.41(3.76)$ \\
\hline Duration of SJIA-years (SD) & $6.08(3.94)$ \\
\hline Duration of refractory sJIA-years (SD) & $4.26(3.21)$ \\
\hline \multicolumn{2}{|l|}{ Patients' conditions at onset of refractory SJIA } \\
\hline \multicolumn{2}{|l|}{ Clinical manifestations } \\
\hline Systemic features - no (\%) & $15(34.88)$ \\
\hline Arthritis-no (\%) & $29(67.44)$ \\
\hline \multicolumn{2}{|l|}{ Steinbrocker's Functional Class } \\
\hline Class I/II-no (\%) & $37(86.04)$ \\
\hline Class III/IV-no (\%) & $6(13.96)$ \\
\hline \multicolumn{2}{|l|}{ Health insurance schemes } \\
\hline Universal coverage scheme-no (\%) & $39(90.70)$ \\
\hline Civil Servant Medical Benefit Scheme-no (\%) & $1(2.33)$ \\
\hline Patient self-payment-no (\%) & $1(2.33)$ \\
\hline Disability benefits-no (\%) & $2(4.64)$ \\
\hline \multicolumn{2}{|l|}{ No of events* } \\
\hline Standard treatment alone-total no (\%) & $122(100)$ \\
\hline Active disease to inactive disease - no (\%) & $67(54.92)$ \\
\hline Inactive disease to active disease-no (\%) & $53(43.44)$ \\
\hline Inactive disease to remission-no (\%) & $2(1.64)$ \\
\hline $\begin{array}{l}\text { Standard treatment plus tocilizumab - total } \\
\text { no }(\%)\end{array}$ & $88(100)$ \\
\hline Active disease to inactive disease - no (\%) & $46(52.27)$ \\
\hline Inactive disease to active disease-no (\%) & $40(45.45)$ \\
\hline Inactive disease to remission - no (\%) & $2(2.27)$ \\
\hline
\end{tabular}

*There is no progress from remission to active disease according to the medical record review.

sJIA, systemic juvenile idiopathic arthritis.

comprised 12597816 people in $2017 .^{32}$ Due to the lack of information, the epidemiological data for JIA applied in this study were from Oman, which is also an Asian country. The prevalence and incidence rates for JIA were $0.02 \%$ and $0.002 \%$ per year, respectively. The proportion of sJIA to JIA in Thailand was $33.8 \%$ according to a retrospective study from 1997 to 2012, ${ }^{12}$ while the proportion of refractory sJIA was around $20.09 \%$ based on our data collection from seven tertiary hospitals. Thus, the number of patients with refractory sJIA was 171 cases in 2017, with 17 new cases annually.

\section{Model structure}

The model stimulated the progression of patients with sJIA with active disease (AC) to inactive disease (IN), remission (RM) and death with lifetime horizon and 3-month cycle length. Patients transitioned to different health states depended on their clinical symptoms (systemic features and/or arthritis), laboratory results (erythrocyte 


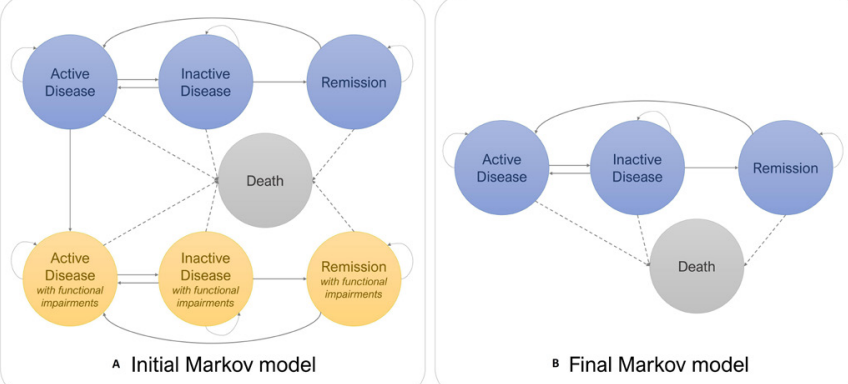

Figure 1 Markov model structure.

sedimentation rate and/or C-reactive protein) and medication status (on or off medications) as shown in the online supplementary table S2. Additionally, the Wallace criteria for inactive disease and clinical remission were applied to the definitions of inactive disease and remission health states (see details on the Wallace criteria in online supplementary table S3). ${ }^{33}$ Both patients with inactive disease and remission had normal laboratory results and no clinical symptoms, but their medication status and period of medication discontinuation were different. Particularly, in case of on medication or off medication for less than 1 year, patients would be defined as inactive disease, while patients with remission had to stop medication for at least 1 year. Stakeholders initially suggested including long-term effects, which are functional impairments indicating by irreversible Steinbrocker's functional class III/IV, defined as limited to little or none of the duties of usual occupation or self-care for class III, and incapacitated, largely or wholly bed-ridden or confined to a wheelchair with little or no self-care for class IV (figure 1A). Nevertheless, the data availability was not adequate for analysing this factor, that is, only three patients with irreversible status of Steinbrocker's functional class III/IV were found during the data collection period. Finally, the health state with functional impairments was removed (figure 1B).

\section{Model assumptions}

The following assumptions were applied to the Markov model.

1. A parametric survival analysis was employed to extrapolate survivor function beyond the period observed in this study in order to estimate transitional probabilities for a life-time horizon.

2. Since the mortality rate of refractory sJIA is inconclusive, the probability of death due to this disease was adopted for the active disease health state only. For IN and RM health states, the mortality does not differ from the general population in Thailand.

3. The probability of relapse would begin after the first 5 years of remission as it conforms to data from our systematic review and expert consensus in stakeholders' meetings.

4. Owing to the inadequate number of patients with refractory sJIA, direct non-medical costs and utility scores were assumed to be equal to those of patients with general sJIA who were in the same health state (figure 1A).

\section{Model inputs}

All model inputs including transitional probabilities, cost variables and utility scores are shown in tables 2 and 3 . The transitional probabilities and direct medical costs were derived from the data from patients with refractory sJIA, while direct non-medical costs and utility scores were collected from both patients with general sJIA and patients with refractory sJIA.

\section{Transitional probabilities}

Transitional probabilities were required for the Markov model to simulate the events of patients entering each health state and were generated using data of clinical conditions from medical records of 43 patients with refractory sJIA (table 1 ). These data were collected as a retrospective comparative study (level III), ${ }^{34}$ which was compared between two arms, that is, standard treatment alone and standard treatment plus tocilizumab. A parametric survival analysis was then applied and parametric distributions were used to describe the time to event, including Exponential, Weibull and Gompertz distributions according to the fitting model using Akaike information criterion, Bayesian information criterion and graphical visualisation (table 2). The formulas for each distribution are available in the online supplementary table S4. Particularly, the coefficient for the covariate of patient's age at refractory disease onset (age coefficient) and constant for baseline hazard were related to lambda, but the coefficient was taken into account only if statistically significant. Besides this, the probability of transition from remission to active disease (relapse) was derived from a systematic review (see online supplementary tables S5 and S6) because there were no data available for patients in a remission state turning to an active disease state according to the medical record review. The probability of death due to other causes was estimated from the WHO's 2015 life table for Thailand. ${ }^{35}$

\section{Cost variables}

A social perspective was applied in this study. The cost components included direct medical and non-medical costs in the US dollar (USD). Direct medical costs include diagnosis and treatment costs. The price of tocilizumab was quoted from the National Drug Information under the Thai Food and Drug Administration. ${ }^{36}$ The cost of one course of tocilizumab treatment was calculated according to the model option. Apart from the cost of a new intervention, the cost of medicines and other services for both refractory sJIA and other conditions (ie, complications of refractory sJIA and adverse events of treatment) were covered. The cost of medicines was divided into two groups. The first group comprised cost of standard medicines or standard treatment, which included NSAIDs, systemic corticosteroids and nonbiological DMARDs. The second group consisted of the 


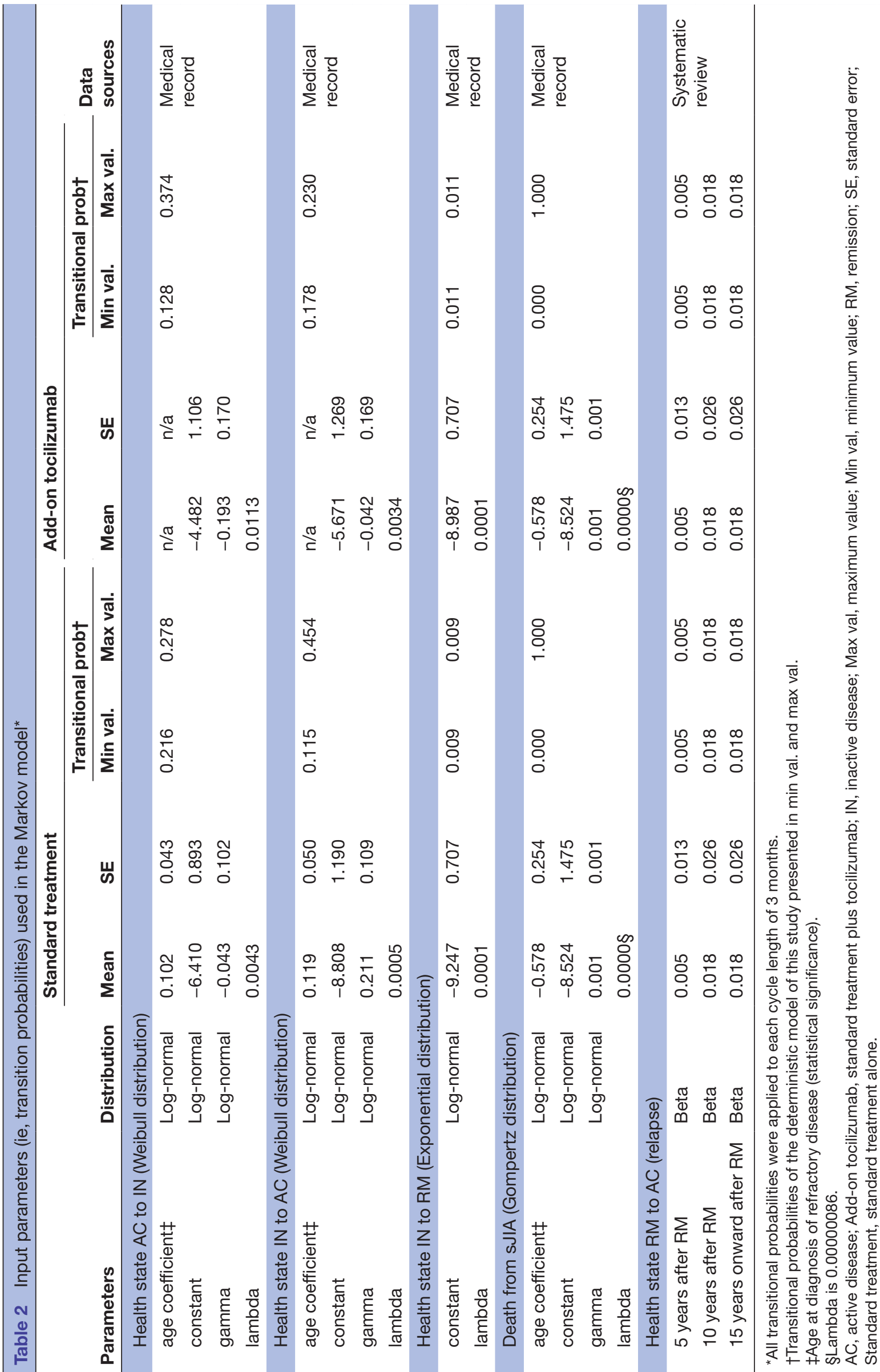


Table 3 Input parameters (ie, costs and utility parameters) used in the Markov model ${ }^{*}$

\begin{tabular}{|c|c|c|c|c|c|c|}
\hline \multirow[b]{2}{*}{ Parameters } & \multirow[b]{2}{*}{ Distribution } & \multicolumn{2}{|c|}{$\begin{array}{l}\text { Standard } \\
\text { treatment }\end{array}$} & \multicolumn{2}{|c|}{$\begin{array}{l}\text { Add-on } \\
\text { tocilizumab }\end{array}$} & \multirow[b]{2}{*}{ Data sources } \\
\hline & & Mean & SE & Mean & SE & \\
\hline \multicolumn{7}{|l|}{ Direct medical costs (USD) } \\
\hline Cost of tocilizumab (TCZ) & & & & & & Regimen: \\
\hline $\begin{array}{l}\text { Price of TCZ } 80 \mathrm{mg} / 4 \mathrm{~mL} \text { per one vial } \\
\text { Cost of TCZ per one course }\end{array}$ & - & - & - & 162 & - & $\begin{array}{l}\text { Stakeholders' consensus } \\
\text { TCZ's price: } \\
\text { Thai FDA }\end{array}$ \\
\hline TCZ IV every 2 weeks for 6 months & - & - & - & 7778 & - & \\
\hline TCZ IV every 3 weeks for 3 months & - & - & - & 2593 & - & \\
\hline TCZ IV every 4 weeks for 3 months & - & - & - & 1945 & - & \\
\hline TCZ IV every 6 weeks for 3 months & - & - & - & 1296 & - & \\
\hline TCZ IV every 8 weeks for 6 months & - & - & - & 1945 & - & \\
\hline Total cost & - & - & - & 15556 & - & \\
\hline Cost of standard medicines $†$ & & & & & & Medical record \\
\hline Health state AC (IPD) & Gamma & 68 & 57 & 8 & 4 & \\
\hline Health state $A C(O P D)$ & Gamma & 45 & 10 & 46 & 16 & \\
\hline Health state IN (OPD) & Gamma & 46 & 12 & 51 & 46 & \\
\hline Cost of other medicines $\ddagger$ & & & & & & Medical record \\
\hline Health state AC (IPD) & Gamma & 85 & 54 & 64 & 23 & \\
\hline Health state AC (OPD) & Gamma & 10 & 2 & 58 & 27 & \\
\hline Health state IN (OPD) & Gamma & 100 & 90 & 13 & 7 & \\
\hline Cost of services & & & & & & Medical record \\
\hline Health state AC (IPD) & Gamma & 276 & 65 & 276 & 65 & \\
\hline Health state $A C(O P D)$ & Gamma & 61 & 7 & 61 & 7 & \\
\hline Health state IN (OPD) & Gamma & 63 & 13 & 63 & 13 & \\
\hline Health state RM (OPD) & Gamma & 5 & 0 & 5 & 0 & \\
\hline \multicolumn{7}{|l|}{ Direct non-medical costs (USD)§ } \\
\hline Total cost for 1 OPD visit & Gamma & 53 & 7 & 53 & 7 & Interview \\
\hline Total cost for 1 IPD visit & Gamma & 60 & 10 & 60 & 10 & \\
\hline Extra cost for the first yearq & Gamma & 17 & 6 & 17 & 6 & \\
\hline \multicolumn{7}{|l|}{ Hospital visits } \\
\hline Frequency of IPD visits per 3 months & & & & & & Medical record \\
\hline Health state $A C$ & Gamma & 0.04 & 0.01 & 0.40 & 0.01 & \\
\hline Duration between each OPD visit (days) & & & & & & Medical record \\
\hline Health state $A C$ & Gamma & 45.22 & 4.43 & 34.93 & 6.09 & \\
\hline Health state IN & Gamma & 58.58 & 3.79 & 48.77 & 9.49 & \\
\hline Health state RM & Gamma & 204.75 & 36.75 & 204.75 & 36.75 & \\
\hline \multicolumn{7}{|l|}{ Utility parameters } \\
\hline Health state $A C(n=31)$ & Gamma & 0.714 & 0.051 & 0.714 & 0.051 & Interview \\
\hline Health state IN $(n=50)$ & Gamma & 0.886 & 0.021 & 0.886 & 0.021 & \\
\hline Health state $\mathrm{RM}(\mathrm{n}=8)$ & Gamma & 0.937 & 0.042 & 0.937 & 0.042 & \\
\hline
\end{tabular}

Continued 


\begin{tabular}{|c|c|c|c|c|c|c|}
\hline \multirow[b]{2}{*}{ Parameters } & \multirow[b]{2}{*}{ Distribution } & \multicolumn{2}{|c|}{$\begin{array}{l}\text { Standard } \\
\text { treatment }\end{array}$} & \multicolumn{2}{|c|}{$\begin{array}{l}\text { Add-on } \\
\text { tocilizumab }\end{array}$} & \multirow[b]{2}{*}{ Data sources } \\
\hline & & Mean & SE & Mean & SE & \\
\hline
\end{tabular}

${ }^{*}$ All costs in the year 2018 (US $\$ 1=$ THB31.20 in February 2020).

†Standard medicines (standard treatment) including NSAIDs (eg, naproxen, ibuprofen and indomethacin), systemic corticosteroids (eg, prednisolone and methylprednisolone) and non-biological DMARDs (eg, sulfasalazine, methotrexate and hydroxychloroquine).

$\ddagger$ Other medicines including medicines for macrophage activation syndrome, severe infections (ie, sepsis and pneumonia) and eye problems (ie, cataracts and glaucoma).

§Direct non-medical costs including additional food and travel expenses of patients and parents, and income loss of parents.

IExtra cost for the first year including cost of house renovation, wheelchairs and patient beds.

AC, active disease; Add-on tocilizumab, standard treatment plus tocilizumab; DMARD, disease-modifying antirheumatic drug; FDA, Food and Drug Administration; IN, inactive disease; IPD, inpatient department; IV, intravenous; NSAID, non-steroidal anti-inflammatory drug; OPD, outpatient department; RM, remission; SE, standard error; Standard treatment, standard treatment alone; USD, US dollar.

cost of other medicines which were used for the treatment of other conditions as mentioned above, including MAS, severe infections (ie, sepsis and pneumonia) and eye problems (ie, cataracts and glaucoma). Nonetheless, some specific adverse events of tocilizumab, especially infections, were not separately considered in this study because patients with tocilizumab treatment also received high-dose corticosteroids and DMARDs. Thus, the cause of infection could not be isolated to tocilizumab alone. The cost variables were collected from four tertiary hospital databases based on their willingness and were in the form of charges that were converted to costs using the ratio of cost to charge of $1.63 .^{25}$ Moreover, direct medical costs were converted to cost of the year 2018 by using the Thai consumer price index ${ }^{37}$ and categorised by treatment options, health states and hospital departments (ie, inpatient department (IPD) and outpatient department (OPD) (table 3). As for the direct non-medical costs, including food, travel and accommodation expenditures, the data were obtained through face-to-face interviews with caregivers at seven tertiary hospitals $(n=89)$ and categorised by hospital departments as well. In addition, the cost of each OPD and IPD visit was multiplied by the number of IPD and OPD visits to calculate the direct cost per one cycle (3 months).

\section{Utility scores}

Primary data collection was performed at seven hospitals to estimate the health utility weights $(0=$ death and $1=$ full health) of each health state. These utility scores were measured using EQ-5D-3L (EuroQoL registration ID 26707; EuroQoL, Rotterdam, the Netherlands), which consisted of five dimensions including mobility, self-care, usual activities, pain/discomfort and anxiety/depression. ${ }^{38}$ The caregiver was interviewed to rate the patient's health-related QoL instead of the patients themselves because the target population of this study was patients who were $\geq 2$ years old $(n=89)$. On the same day of the caretaker interview, physicians assessed patients' disease activity and complications to identify their health states as well. Utility was then derived from the Thai EQ-5D-3L value set. ${ }^{39}$ However, utility scores were not calculated separately according to treatment groups due to the limited number of patients during the primary data collection period. Therefore, the utility score values were 0.714 for active disease, 0.886 for inactive disease and 0.937 for remission (table 3 ). As for disutility due to complications and adverse events, it was not taken into account because there was no difference between two groups owing to the small number of patients in this study, and the causes of complications and adverse events could not be differentiated as mentioned in the 'cost variables' session.

\section{Uncertainty analysis}

One-way and probabilistic sensitivity analysis were conducted to investigate parameter uncertainty. In the one-way sensitivity analysis, model parameters, such as costs, utilities and visit numbers, were adjusted by a 95\% CI according to the Bayesian interval method. The results of the one-way sensitivity analysis were presented in a tornado diagram. As for the probabilistic sensitivity analysis, a Monte Carlo simulation was run 5000 times using Microsoft Excel (Microsoft). Random sampling of the model parameters were based on data distribution (tables 2 and 3): (1) beta distribution for transitional probabilities and utility scores because the values were ranged $0-1$; (2) gamma distribution for cost variables because these parameters must be positive values and (3) log-normal distribution for survival parameters. The results were presented as a cost-effectiveness acceptability curve.

\section{Budget impact analysis}

The budget impact analysis was employed to predict the potential financial impact of the adoption of tocilizumab as add-on therapy for the first 5 years using a governmental perspective; thus, only direct medical costs were considered. The total number of patients with refractory sJIA in Thailand was estimated using epidemiological data to obtain the aforementioned target population. Additionally, various percentages of access to healthcare services, including $100 \%, 50 \%$ and $25 \%$, were adjusted according to the maximum possibility, the expert consensus in stakeholders' meetings ${ }^{162}$ and the medical record review, respectively. 


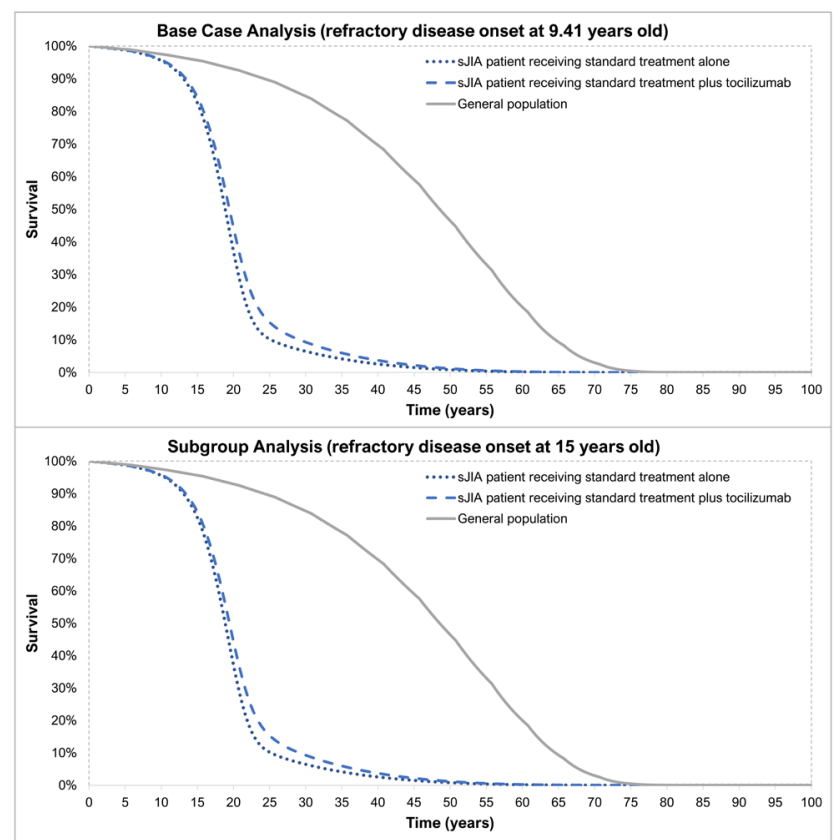

Figure 2 Survival prediction modelling for base case and subgroup analysis. SJIA, systemic juvenile idiopathic arthritis.

\section{Patient and public involvement}

No patient involved.

\section{RESULTS}

\section{Model validation}

The model structure, inputs and outputs were assessed by face validity in the stakeholders' meeting for consideration of the preliminary results. ${ }^{16} 28$ The predicted survival curve of patients with refractory sJIA receiving standard treatment alone compared with those receiving tocilizumab as an add-on therapy to standard treatment and the general population was shown in figure 2 for both the base case and subgroup analysis.

\section{Cost-utility analysis}

The model simulated the lifetime of patients with refractory sJIA for the base case. The total lifetime costs and QALYs of using standard treatment alone and adding tocilizumab to standard treatment are shown in table 4 . The ICER was US\$35 799 per QALY gained, which was not cost-effective compared with the Thai cost-effectiveness threshold of US $\$ 5128$ per QALY gained. In the subgroup analysis, an increase in ICER could be seen in the group of disease occurring at 15 years old, accounting for US\$53301 per QALY gained.

\section{Uncertainty analysis}

In the one-way sensitivity analysis, the most influential parameter was the discount rate for outcome followed by the price of tocilizumab $80 \mathrm{mg} / 4 \mathrm{~mL}$ for one vial and the transitional probability of relapse after remission for 15 years onward. However, there was no parameter range that could change ICER to the cost-effective result or values $<$ US\$5128 per QALY gained (figure 3).

In the probabilistic sensitivity analysis, the costeffectiveness acceptability curves representing the probability of both treatment options at different ceiling thresholds for cost-effectiveness are indicated in figure 4 . At the cost-effectiveness threshold of US $\$ 5128$ per QALY gained, the current standard treatment could become cost-effective (95\%). However, if the threshold was US\$35 799 per QALY gained, both treatment options were potentially equally cost-effective. Additionally, the results of subgroup analysis showed that if the patients receiving tocilizumab as add-on therapy were 15 years old, the possibility of new interventions to be cost-effective at the cost-effectiveness threshold would decrease compared with standard treatment.

Table 4 Results of costs, health outcomes and ICER of using tocilizumab as add-on therapy to standard treatment compared with standard treatment alone*

\begin{tabular}{|c|c|c|c|c|}
\hline Options & $\begin{array}{l}\text { Total cost } † \\
\text { (USD) }\end{array}$ & $\begin{array}{l}\text { Life years } \\
\text { (years) }\end{array}$ & $\begin{array}{l}\text { QALYst } \\
\text { (years) }\end{array}$ & $\begin{array}{l}\text { ICER } \\
\text { (USD/QALY gained) }\end{array}$ \\
\hline \multicolumn{5}{|c|}{ Base case analysis (refractory disease onset at 9.41 years old) } \\
\hline Add-on tocilizumab & 47139 & 31.98 & 13.16 & \\
\hline Incremental value & 26592 & 1.55 & 0.74 & \\
\hline Add-on tocilizumab & 59765 & 43.28 & 15.53 & \\
\hline Incremental value & 34697 & 1.32 & 0.65 & \\
\hline
\end{tabular}

*The results were obtained from probabilistic model stimulating 5000 times.

†Discounted values.

‡Undiscounted values, life year at birth.

Add-on tocilizumab, standard treatment plus tocilizumab; ICER, incremental cost-effectiveness ratio; QALYs, quality-adjusted life years;

Standard treatment, standard treatment alone; USD, US dollar. 


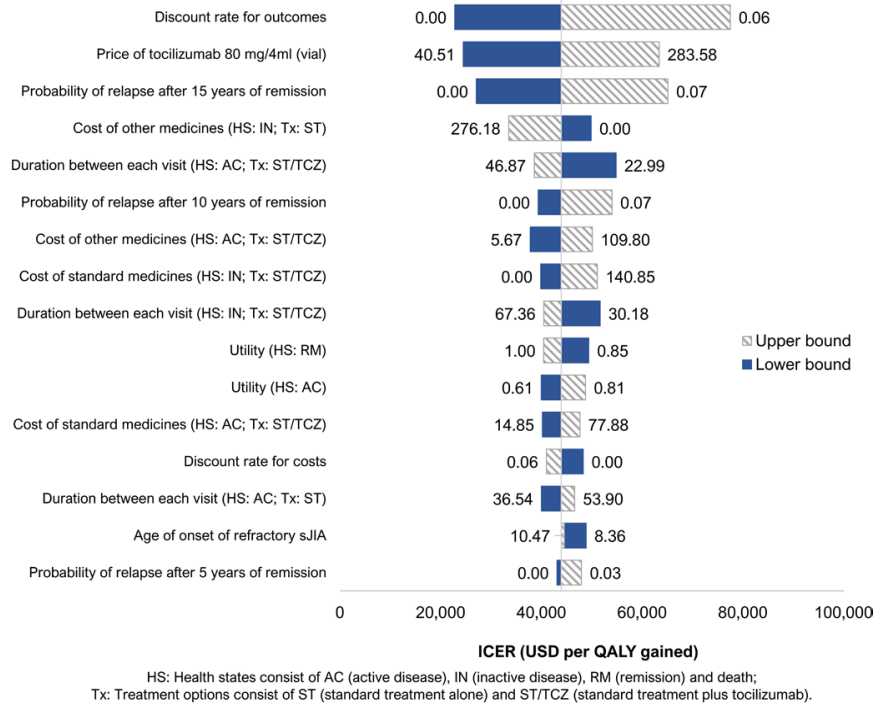

Figure 3 Tornado graph showing the results of one-way sensitivity analysis. This figure indicates the parameters with the largest effect on ICER (USD per QALY gained) when they are varied individually. ICER, incremental cost-effectiveness ratio; QALY, quality-adjusted life-year; USD, US dollar.

\section{Budget impact analysis}

The estimated number of Thai patients with refractory sJIA was 171 cases, with an incidence rate of 17 cases per year. However, the data from medical records indicate that the current access rate to healthcare services was approximately 25\%. Meanwhile, the stakeholders predicted

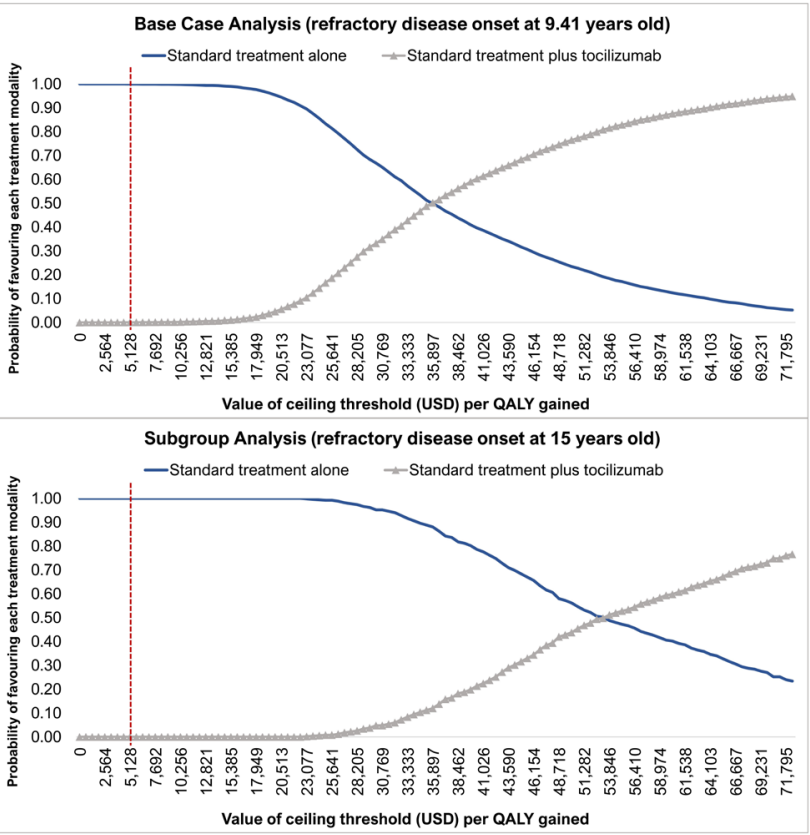

Figure 4 Cost-effective acceptability curve of base case and subgroup analysis. This shows the probabilities of each option being cost-effective at a given ceiling ratio. The dashed line represents the cost-effectiveness thresholds of 5128 USD per QALY gained in the adoption of health interventions in Thailand. QALY, quality-adjusted life-year; USD, US dollar. that this access rate might reach $50 \%$ after tocilizumab is included in the NLEM.

The total budget for each treatment option in all scenarios was illustrated in table 5 . To provide tocilizumab as add-on treatment for every patient ( $100 \%$ access rate), the Thai government must allocate an additional budget of approximately US $\$ 2.5$ million for the first year, which is higher than for other years due to the number of patients based on the prevalence and frequency of tocilizumab given in the first year. The additional budget could then be decreased to around US $\$ 0.9$ million for the second year and around US $\$ 0.5$ million for the last 3 years. Thus, the budget impact would be US $\$ 4.8$ million for the 5 -year budget impact or around US $\$ 1$ million for the annual average budget impact. In addition, other scenarios including $25 \%$ and $50 \%$ access rates led to a lower additional budget impact, that is, US $\$ 1.4$ and US $\$ 2.4$ million of 5-year budget impacts, respectively.

\section{DISCUSSION}

This study was the first economic evaluation of tocilizumab use in a refractory sJIA and multicentre study that collected data from tertiary hospitals where paediatric rheumatologists were providing treatment for patients with sJIA in Thailand.

The results indicated that using tocilizumab as an add-on therapy for refractory sJIA was not cost-effective according to the ICER of US\$35799 per QALY gained, which was higher than the Thai cost-effectiveness threshold of US\$5128 per QALY gained. Investing in this health technology requires an additional budget of around US\$1 million per year to cover $100 \%$ of the patients with refractory sJIA. In addition, the first three parameters affecting the cost-effectiveness results were the discount rate for outcome, the price of tocilizumab $80 \mathrm{mg} / 4 \mathrm{~mL}$ and the transitional probability of relapse after remission for $\geq 15$ years.

According to our systematic review, five studies of economic evaluations of tocilizumab used for sJIA treatment were found in conference proceedings, ${ }^{18-22}$ but no full articles were available (see online supplementary tables S7 and S8). All of these studies showed the costeffectiveness of tocilizumab in their countries. The target population of these studies were sJIA or refractory sJIA, but the interventions and comparative factors varied according to the different clinical practice guidelines for each country. In four of the five studies, ${ }^{18-20} 22$ tocilizumab was used alone or followed by other biological agents, such as etanercept, adalimumab and abatacept, compared with placebo, methotrexate, prednisolone or other biologics. These treatments differ to the Thai clinical practice, which is to use standard treatment including NSAIDs, steroids and DMARDs, with tocilizumab as an add-on therapy. In addition, three of the five studies ${ }^{20-22}$ indicated that the efficacy used in their studies were obtained from the double-blind, placebo-controlled, randomized phase III TENDER trial (WA18221), ${ }^{23}$ and 
Table 5 Estimated total and incremental budgets for base and scenario cases

\begin{tabular}{|c|c|c|c|c|c|c|}
\hline \multirow[b]{2}{*}{ Scenarios } & \multirow[b]{2}{*}{ Year } & \multicolumn{2}{|c|}{ Total budget (USD) } & \multicolumn{3}{|c|}{ Incremental budget (USD) } \\
\hline & & $\begin{array}{l}\text { Standard } \\
\text { treatment }\end{array}$ & $\begin{array}{l}\text { Add-on } \\
\text { tocilizumab }\end{array}$ & $\begin{array}{l}\text { 1-year budget } \\
\text { impact }\end{array}$ & $\begin{array}{l}\text { 5-year budget } \\
\text { impact }\end{array}$ & $\begin{array}{l}\text { Average budget } \\
\text { impact per year }\end{array}$ \\
\hline \multirow{2}{*}{$25 \%$ access } & 2 & 34269 & 303088 & 268819 & & \\
\hline & 3 & 35627 & 160323 & 124697 & & \\
\hline \multirow[t]{4}{*}{$50 \%$ access } & 1 & 93481 & 1339748 & 1246266 & 2440596 & 488119 \\
\hline & 2 & 69625 & 527654 & 458028 & & \\
\hline & 3 & 73036 & 309653 & 236617 & & \\
\hline & 4 & 75318 & 321043 & 245725 & & \\
\hline \multirow{3}{*}{$100 \%$ access } & 3 & 143626 & 597723 & 454097 & & \\
\hline & 4 & 147570 & 619029 & 471459 & & \\
\hline & 5 & 152373 & 639438 & 487066 & & \\
\hline
\end{tabular}

*The results were obtained from a government perspective.

Add-on tocilizumab, standard treatment plus tocilizumab; Standard treatment, standard treatment alone; USD, US dollar.

JIA ACR responses and the absence of fever were used as efficacy outcomes. These outcomes were intermediate and there was no clinical study revealing the final outcomes, such as morbidity and mortality, because the duration of the study was only $48-102$ weeks. ${ }^{23} 24$

There were three main limitations in this study. First, the data from medical records in some hospitals were insufficient to calculate the JIA ACR response while the Thai HTA guidelines recommended deriving the input parameters from the Thai patients' data. Therefore, the stakeholders agreed to use the disease status adapted from Wallace criteria, ${ }^{33}$ including inactive disease and remission. Second, no randomised controlled trial (RCT) matched the outcomes of interest. Therefore, obtaining real-world data was necessary for the effectiveness of tocilizumab as add-on therapy, otherwise its effectiveness would be underestimated because the patients could not access the best standard practice of using tocilizumab due to financial barriers leading to delayed tocilizumab treatment for most patients, resulting in poor outcomes. ${ }^{40}$ Therefore, the results cannot be generalised to all patients with refractory sJIA. Likewise, the inclusion criteria of RCTs were different from those of this study. In RCTs, patients with active systemic JIA for at least a 6-month duration and had inadequate response to NSAIDs and systemic corticosteroids were included. However, patients in this observational study had a longer duration of failure towards the standard treatment. This meant that the clinical conditions of patients in this study were more severe than RCTs. Thus, the results of this study could not be compared with RCTs. ${ }^{23}{ }^{24}$ Third, the initial Markov model containing functional impairments could not be employed due to limited data because only three patients with an irreversible status of Steinbrocker's class III/IV were identified during the data collection period.

\section{POLICY RECOMMENDATIONS}

Internationally, the UK National Health Service provides tocilizumab for patients with sJIA as a benefit under a patient-access scheme, which discounts all invoices for tocilizumab. Meanwhile, the Australian Government's Pharmaceutical Benefits Scheme subsidises tocilizumab treatment for severe active JIA, including sJIA and other subtypes.

In the low-income to middle-income country context, price negotiation is an important approach to increase access to high-cost essential medicines. ${ }^{41}$ Although tocilizumab is not cost-effective at its current price, it is necessary for patients with refractory sJIA because of the absence of an effective treatment in the NLEM. Thus, a price-negotiation process in the development of the Thai NLEM should be performed using rebates, risk-sharing arrangements or managed entry agreements to ensure the affordability of government budget holders in the healthcare system.

Moreover, tocilizumab should be included in a specialaccess programme for NLEM in the government sector because tocilizumab use and health outcomes should be monitored evaluated in a real-world setting. There are three benefits if tocilizumab can be included in this programmes: (1) patients with refractory sJIA can access 
tocilizumab as soon as they are diagnosed, resulting in a better outcome and an improvement in the QoL for both patients and their families ${ }^{40}$; (2) according to the special-access programme requiring monitoring and evaluation, the data collection could be controlled with fewer confounding factors and more homogeneity, which would lead to more reliable efficacy, safety and cost-effectiveness and (3) the NLEM Subcommittee will be able to reconsider efficacy, cost-effectiveness, drug price and affordability if tocilizumab should continue to be available in the NLEM.

\section{Author affiliations}

${ }^{1}$ Health Intervention and Technology Assessment Program, Nonthaburi, Thailand

${ }^{2}$ Division of Rheumatology, Department of Pediatrics, Faculty of Medicine

Ramathibodi Hospital, Mahidol University, Bangkok, Thailand

${ }^{3}$ Division of Rheumatology, Department of Pediatrics, Faculty of Medicine Siriraj Hospital, Mahidol University, Bangkok, Thailand

${ }^{4}$ Division of Allergy, Immunology and Rheumatology, Department of Pediatrics, Queen Sirikit National Institute of Child Health, Bangkok, Thailand

${ }^{5}$ Pediatric Allergy \& Clinical Immunology Research Unit, Department of Pediatrics,

Faculty of Medicine, Chulalongkorn University, Bangkok, Thailand

${ }^{6}$ Department of Pediatrics, Khon Kaen Hospital, Khon Kaen, Thailand

${ }^{7}$ Division of Rheumatology, Department of Medicine, Faculty of Medicine, Khon Kaen University, Khon Kaen, Thailand

${ }^{8}$ Division of Allergy, Immunology and Rheumatology, Department of Pediatrics, Faculty of Medicine, Thammasat University, Pathum Thani, Thailand

Acknowledgements The authors would like to thank Salakjit Chuenchom, Butsabong Lerkvaleekul, Sirisucha Soponkanaporn, Maynart Sukharomana, Sirikarn Tangcheewinsirikul, Tassalapa Daengsuwan, Dara Mairiang, Juthamas Yamsuwan, Thuanchai Seelasana, Thanwatip Thanathornthiansiri and Wanlapa Weerapakorn for their contribution to data collection of this project.

Contributors NK, PL, SV, SC, GP, PK, MT, RN and SN contributed to the conception of the study. NK and PL developed study design and methods. NK, DK, CC, SV, SC, GP, PK, MT, RN and SN contributed to data collection and study site investigation. NK and PL analysed and interpreted data. NK, DK and CC contributed to project administration. PL, SV and SC supervised the overall project. NK, PL, CC and DK drafted the manuscript. NK, PL, SV and SC critically revised the manuscript. NK, PL, DK, CC, SV, SC, GP, PK, MT, RN and SN reviewed and approved for the submitted manuscript.

Funding This work was supported by Bureau of Drug Control, Thai Food and Drug Administration (FDA) grant number 504/2561. Health Intervention and Technology Assessment Program (HITAP) is funded by the Thailand Research Fund under the senior research scholar on Health Technology Assessment (RTA5980011) and the Bureau of Health Policy and Strategy, Ministry of Public Health. HITAP's international unit has been supported by the international Decision Support Initiative (funded by the Bill \& Melinda Gates Foundation and the Department for International Development (DFID), the UK government department responsible for administering overseas aid to promote sustainable development and eliminate world poverty) and the Rockefeller Foundation to provide technical assistance on health intervention and technology assessment for governments of low-income and middle-income countries. The findings, interpretations and conclusions expressed in this article do not necessarily reflect the views of the aforementioned funding agencies.

Competing interests None declared.

Patient consent for publication Not required.

Ethics approval The research was approved by the Committee for Research, Faculty of Medicine Ramathibodi Hospital, Mahidol University (COA No. MURA2018/709), the Ethics Committee for Research in Humans, Faculty of Medicine Siriraj Hospital, Mahidol University (COA No. Si757/2018), the Research Ethics Review Committee of Queen Sirikit National Institute of Child Health (COA No. REC.015/2562), the Research Ethics Review Committee for Research Involving Human Subjects (COA No. 025/2019), Khon Kaen Hospital Institute Review Board in Human Research (Ref. No. KE61118), the Khon Kaen University Ethics Committee in Human Research (Ref. No. HE611543), the Human Research Ethics Committee of Thammasat University (COA No. 230/2561).
Provenance and peer review Not commissioned; externally peer reviewed.

Data availability statement Data may be obtained from a third party and are not publicly available. The data that support the findings of this study are available on request from the corresponding author. The data are not publicly available due to privacy or ethical restrictions.

Open access This is an open access article distributed in accordance with the Creative Commons Attribution Non Commercial (CC BY-NC 4.0) license, which permits others to distribute, remix, adapt, build upon this work non-commercially, and license their derivative works on different terms, provided the original work is properly cited, appropriate credit is given, any changes made indicated, and the use is non-commercial. See: http://creativecommons.org/licenses/by-nc/4.0/.

ORCID iD

Pattara Leelahavarong http://orcid.org/0000-0002-9584-0293

\section{REFERENCES}

1 Petty RE, Southwood TR, Manners P, et al. International League of associations for rheumatology classification of juvenile idiopathic arthritis: second revision, Edmonton, 2001. J Rheumatol 2004;31:390-2.

2 Prakken B, Albani S, Martini A. Juvenile idiopathic arthritis. Lancet 2011;377:2138-49.

3 Lee JJY, Schneider R. Systemic juvenile idiopathic arthritis. Pediatr Clin North Am 2018;65:691-709.

4 Firestein GS, Budd RC, Gabriel SE, et al. Kelley's textbook of rheumatology. 9 edn. Philadelphia: Elsevier Saunders, 2013: 1752-68.

5 Cassidy JT, Petty RE, Laxer RM, et al. Textbook of pediatric rheumatology. 6 edn. Philadelphia: Saunders Elsevier, 2011: 236-48.

6 Petty RE. Prognosis in children with rheumatic diseases: justification for consideration of new therapies. Rheumatology 1999;38:739-42.

7 Spiegel LR, Schneider R, Lang BA, et al. Early predictors of poor functional outcome in systemic-onset juvenile rheumatoid arthritis: a multicenter cohort study. Arthritis Rheum 2000;43:2402-9.

8 Charuvanij S, Chaiyadech C. Health-Related quality of life in children with early-stage juvenile idiopathic arthritis. Musculoskeletal Care 2019;17:215-20.

9 Sontichai W, Vilaiyuk S. The correlation between the childhood health assessment questionnaire and disease activity in juvenile idiopathic arthritis. Musculoskeletal Care 2018;16:339-44.

10 Thierry S, Fautrel B, Lemelle I, et al. Prevalence and incidence of juvenile idiopathic arthritis: a systematic review. Joint Bone Spine 2014;81:112-7.

11 De Benedetti F, Schneider R. Chapter 16 - Systemic Juvenile Idiopathic Arthritis. In: Petty RE, Laxer RM, Lindsley CB, et al, eds. Textbook of pediatric rheumatology. Seventh Edition. Philadelphia: W.B. Saunders, 2016: 205-16.

12 Vilaiyuk S, Soponkanaporn S, Jaovisidha S, et al. A retrospective study on 158 Thai patients with juvenile idiopathic arthritis followed in a single center over a 15-year period. Int $J$ Rheum Dis 2016;19:1342-50.

13 Teerawattananon Y, Tantivess S, Yothasamut J, et al. Historical development of health technology assessment in Thailand. Int $J$ Technol Assess Health Care 2009;25:241-52.

14 The National Drug Committee. National list of essential medicines 2019. Nonthaburi: The Ministry of Public Health, 2019.

15 Hashkes PJ, Laxer RM. Medical treatment of juvenile idiopathic arthritis. JAMA 2005;294:1671-84.

16 Kulpokin D. An economic evaluation of tocilizumab use in patients with systemic juvenile idiopathic arthritis. In: Kittiratchakool N, ed. Stakeholders' meeting on the scope of the study. Meeting Room1, Health Intervention and Technology Assessment Program, 6 th Floor, 6 th Building, Department of Health, Ministry of Public Health, 2018: $1-10$.

17 Income - Expenditure Statistics Group, Social Statistics Bureau, National Statistical Office. The 2015 household socio - economic survey (whole kingdom), 2015. Available: http://web.nso.go.th/en/ survey/house_seco/socio_15_Whole\%20Kingdom.htm [Accessed 20 Jun 2018].

18 Carlos F, Clark P, Lechuga D. PMS39 economic evaluation of tocilizumab for the treatment of systemic juvenile idiopathic arthritis in Mexico. Value in Health 2011;14:A309.

19 Lechuga D, Alva M, Carlos F. PMS28 economic evaluation of the use of anti TNF's and tocilizumab for the treatment of systemic juvenile idiopathic arthritis (sJIA) in Mexico. Value in Health 2012;15:A38. 
20 Hallinen T, Soini EJ, Diamantopoulos A, et al. THU0305 Cost-utility of tocilizumab in the treatment of systemic juvenile idiopathic arthritis in finland. Ann Rheum Dis 2013;71:258.4-9.

21 Vicente C, Sabapathy S, Formica L, et al. Cost-Utility analysis of tocilizumab in the treatment of active systemic juvenile idiopathic arthritis. Value in Health 2013;16:A225.

22 Ryazhenov VV, Gorokhova SG, Emchenko IV. Pharmacoeconomic evaluation of treatment with tocilizumab in Russian children with systemic juvenile idiopathic arthritis. Value Health 2014;17:A380.

23 De Benedetti F, Brunner HI, Ruperto N, et al. Randomized trial of tocilizumab in systemic juvenile idiopathic arthritis. N Engl J Med 2012;367:2385-95.

24 Yokota S, Imagawa T, Mori M, et al. Efficacy and safety of tocilizumab in patients with systemic-onset juvenile idiopathic arthritis: a randomised, double-blind, placebo-controlled, withdrawa phase III trial. Lancet 2008;371:998-1006.

25 Chaikledkaew U, Kittrongsiri K. Guidelines for health technology assessment in Thailand (second edition)--the development process. J Med Assoc Thai 2014;97:S4-9.

26 Perez RCU, Youngkong S, Tantivess S, et al. Health technology assessment process guidelines. Nonthaburi,Thailand: Health Intervention and Technology Assessment Program (HITAP), 2012.

27 Leelahavarong P, Doungthipsirikul S, Kumluang S, et al. Health technology assessment in Thailand: institutionalization and contribution to healthcare decision making: review of literature. Int $J$ Technol Assess Health Care 2019;35:467-73.

28 Kulpokin D, Chanjam C, Chuenchom S. An economic evaluation of tocilizumab use in patients with systemic juvenile idiopathic arthritis. In: Kittiratchakool N, ed. Stakeholders' meeting on the preliminary results of the study. Meeting Room1, Health Intervention and Technology Assessment Program, 6 th Floor, 6 th Building, Department of Health, Ministry of Public Health, 2019: 1-7.

29 Chittchang U. Guideline for growth assessment in Thai children. Nonthaburi: Bureau of Nutrition (BoN), Department of Health, Ministry of Public Health, 2000.

30 Colbert RA. Classification of juvenile spondyloarthritis: Enthesitisrelated arthritis and beyond. Nat Rev Rheumatol 2010;6:477-85.
31 Thai Food and Drug Administration. Searching for product information, 2018. Available: https://porta.fda.moph.go.th/FDA SEARCH_ALL/MAIN/SEARCH_CENTER_MAIN.aspx [Accessed 30 Jun 2019].

32 National Statistical Office. Number of population from registration by age, sex and Province, 2017. Available: http://statbbi.nso.go.th/ staticreport/page/sector/th/01.aspx [Accessed 20 May 2019].

33 Wallace CA, Ruperto N, Giannini E, et al. Preliminary criteria for clinical remission for select categories of juvenile idiopathic arthritis. $J$ Rheumatol 2004;31:2290.

34 Marx RG, Wilson SM, Swiontkowski MF. Updating the assignment of levels of evidence. J Bone Joint Surg Am 2015;97:1-2.

35 World Health Organization. Life tables by country: Thailand, 2018. Available: http://apps. who.int/gho/data/?theme=main\&vid=61640 [Accessed 30 Jun 2019].

36 National Drug Information, Bureau of Drug Control, The Food and Drug Administration. The National medicine systems development Committee announcement: median price 2018, 2018. Available: http://ndi.fda.moph.go.th/drug_value [Accessed 1 May 2018].

37 Bureau of Trade and Economic indices, Ministry of Commerce. 2018 General consumer price index of country, 2018. Available: http:// www.price.moc.go.th/price/cpi/index_new.asp [Accessed 25 Jun 2018].

38 EuroQol Research Foundation. EQ-5D-3L 2018, 2018. Available: https://euroqol.org/eq-5d-instruments/eq-5d-3l-about/ [Accessed 20 June 2018].

39 Tongsiri S, Cairns J. Estimating population-based values for EQ-5D health states in Thailand. Value Health 2011;14:1142-5.

40 Pacharapakornpong T, Vallibhakara SA-O, Lerkvaleekul B, et al. Comparisons of the outcomes between early and late tocilizumab treatment in systemic juvenile idiopathic arthritis. Rheumatol Int 2017;37:251-5

41 Teerawattananon $\mathrm{Y}$, Tritasavit N, Suchonwanich N, et al. The use of economic evaluation for guiding the pharmaceutical reimbursement list in Thailand. Z Evid Fortbild Qual Gesundhwes 2014;108:397-404. 